\title{
Acute Care for Elders Components of Acute Geriatric Unit Care: Systematic Descriptive Review
}

Mary T. Fox, PhD, ${ }^{\text {a }}$ Souraya Sidani, PhD, ${ }^{\mathrm{b}}$ Malini Persaud, PhD, ${ }^{\mathrm{a}}$ Deborah Tregunno PhD, ${ }^{\mathrm{a}}$ Ilo Maimets, MSc, M.I.St., ${ }^{\mathrm{c}}$ Dina Brooks, PhD, ${ }^{\mathrm{d}}$ Kelly O’Brien, $\mathrm{PhD},{ }^{\mathrm{d}}$

${ }^{a}$ Faculty of Health, School of Nursing, York University, ${ }^{b}$ School of Nursing, Ryerson University, ${ }^{\mathrm{c}}$ Steacie Science and Engineering Library, York University, ${ }^{\mathrm{d}}$ Department of Physical Therapy and Graduate Department of Rehabilitation Sciences, University of Toronto

Corresponding Author: Mary Fox, Associate Professor, Faculty of Health, School of Nursing, York University, HNES Building, 4700 Keele St., Toronto, Ontario, Canada, M3J 1P3, email: maryfox@yorku.ca, Tel: 416-736-2100 ext. 23088, Fax: 416-736-5714

Alternate Corresponding Author: Malini Persaud, Assistant Professor, Faculty of Health, School of Nursing, York University, HNES Building, 4700 Keele St., Toronto, Ontario, Canada, M3J 1P3, email: malinip@yorku.ca, Tel: 416-736-2100 ext. 40872, Fax: 416-736-5714

Financial support provided by Canadian Institutes of Health Research (CIHR) grant \#KRS94307. Mary Fox was supported by an Ontario Ministry of Health \& Long-Term Care Career Scientist Award, Kelly O’Brien by a CIHR Fellowship, Souraya Sidani and Dina Brooks by Canada Research Chairs, and Deborah Tregunno by an Ontario Ministry of Health and LongTerm Care Senior Nurse Research Award while conducting this study.

The definitive version is available at www.wiley-synergy.com 


\section{Structured Abstract}

OBJECTIVES. To describe the Acute Care for Elders (ACE) model components implemented as part of acute geriatric unit care and explore the association of each ACE component with outcomes of reduced iatrogenic complications, functional decline, length of hospital stay, nursing home discharges, and costs and increased discharges home.

DESIGN. Systematic descriptive review of 32 articles, including 14 trials reporting on the implementation of ACE components or the effectiveness of their implementation in improving outcomes. Mean effect sizes (ESs) were calculated using trial outcome data. Information describing implementation of the ACE components in the trials was analyzed using content analysis.

SETTING. Acute care geriatric units.

PARTICIPANTS. Acutely ill or injured adults $(\mathrm{N}=6,839)$ with an average age of 81 years. INTERVENTIONS. Acute geriatric unit care was characterized by the implementation of one or more ACE components: medical review, early rehabilitation, early discharge planning, prepared environment, patient-centered care.

MEASUREMENTS. Falls, pressure ulcers, delirium, functional decline, length of hospital stay, discharge destination (home or nursing home), and costs.

RESULTS. Medical review, early rehabilitation, and patient-centered care, characterized by the implementation of standardized and individualized function-focused interventions, had larger standardized mean ESs (all ES = .20) averaged across all outcomes, than did early discharge planning $(\mathrm{ES}=.17)$ or prepared environment $(\mathrm{ES}=.11)$.

CONCLUSION. Specific ACE component interventions of medical review, early rehabilitation, and patient-centered care, appear to be optimal for overall positive outcomes. These findings can 
help service-providers design and evaluate the most effective ACE model within the contexts of their respective institutions to improve outcomes for acutely ill or injured older adults.

Key words: ACE model, elderly, descriptive systematic review, component analysis, outcomes 
During hospitalization for an acute illness or injury, older adults are at risk of experiencing iatrogenic complications and functional decline. ${ }^{1}$ These adverse events have been associated with increased costs, institutionalization, and fatality in this patient population. ${ }^{2,3}$ Consequently, preventing adverse events during hospitalization is a priority to service-providers. ${ }^{1}$ The Acute Care for Elders (ACE) model is a pre-habilitation, ${ }^{4}$ function-focused ${ }^{5}$ approach to the hospital care of older adults that is designed to address these concerns. ${ }^{6}$ The results of a recent metaanalysis demonstrate that acute geriatric unit care, in which the ACE model was implemented to varying degrees during the acute phase of an illness or injury, has significant beneficial effects for improving patient and system level outcomes. ${ }^{7}$ A synthesis of ACE components could facilitate service-providers to implement the ACE model accurately and to reproduce positive outcomes in the practice setting. Because there may be barriers within hospitals, to implementing the full ACE model with fidelity, exploring which components are related to positive outcomes could help service providers to prioritize specific ACE components.

The aims in this systematic descriptive review were to describe ACE components implemented as part of acute geriatric unit care in terms of objectives, ACE interventions, dose, and approach, and to explore the association of each ACE component with the outcomes of reduced iatrogenic complications, functional decline, length of hospital stay, nursing home discharges, and costs and increased discharge home. 


\section{METHODS}

This was a systematic descriptive review of the trials reported in a recent meta-analysis. ${ }^{7}$ For the first aim, conceptual, clinical, and empirical articles that provided accounts of how the ACE components were designed or implemented in these trials were reviewed. For the second aim, trial outcome data were used to calculate the mean effect size (ES) associated with each ACE component. Components associated with larger ESs were interpreted as having larger contributions to the outcomes.

\section{Selection Criteria}

Selection criteria for trials included in this descriptive review have been previously reported. ${ }^{7}$ Conceptual, clinical, and empirical articles that contained supplemental information about one or more ACE components implemented in a trial and described the design of the components or the protocol for implementing them were also included. Eligibility was confirmed when the article cited the included trial or the included trial cited the article as providing additional information about the component(s), or when the authors confirmed that the article provided information about the component(s).

\section{Search Strategy and Study Selection}

In addition to the search strategy previously described, searches were conducted of the names of all authors involved in the trials included in a recent meta-analysis. ${ }^{7}$ Two reviewers independently screened the abstracts of the retrieved citations for potential inclusion. Disagreements about the eligibility of abstracts were resolved by discussion and consensus. Where consensus could not be reached, a third team member independently reviewed the abstract 
and determined final inclusion. When needed, the complete article was retrieved and reviewed to determine eligibility.

\section{Data Extraction}

Relevant data from each included trial and article were extracted and entered onto a previously developed and pilot- tested standardized data extraction form. Information categories included:

- The five ACE components and their respective interventions consisting of medical review (interventions directed at minimizing the detrimental effects of medical treatments on functioning),early rehabilitation (occupational or physical rehabilitation interventions focused on rehabilitating functional abilities), early discharge planning (interventions that address

discharge needs), prepared environment(physical environmental modifications that promote functioning),patient-centered care (predominantly nursing interventions directed at preventing declines in physical, cognitive, and psychosocial status). The five ACE components and their respective interventions were based on previously established definitions. $^{8-10}$

- The objectives of each ACE component.

- The dose at which the ACE components were given, defined according to the time the component was initiated, the frequency with which it was provided, and the duration for which it was provided.

- The approach used in providing each ACE component and its respective interventions, categorized as standardized, individualized, or mixed. Standardized components and interventions were provided to all patients or their families or caregivers. Individualized components and interventions were provided based on the individual needs of patients and 
their families or caregivers. A mixed approach was one in which some interventions within a component were provided, and others were provided based on need.

- The outcomes consisting of iatrogenic complications (falls, pressure ulcers, delirium), functional decline, discharge destination (home, nursing home), length of hospital stay, and costs. Falls were defined according to the number of participants who had one or more falls during their hospital stay. ${ }^{7}$ Pressure ulcers were defined by the number of participants who developed skin breakdown during their hospital stay. ${ }^{7}$ Delirium was defined by the number of participants who experienced one or more delirium episodes during their hospital stay. ${ }^{7}$

Functional decline referred to loss of independence at discharge in one or more of five basic activities of daily living performed 2 weeks before hospital admission. ${ }^{4}$ Discharge destination included discharge from hospital to home (defined as own home or with family) and nursing home (defined as nursing home, sheltered living, or hostel). ${ }^{7}$ Length of hospital stay referred to the total number of days in hospital or, when that was unavailable, to number of days from trial admission to discharge. ${ }^{7}$ Costs were defined by the total hospital costs associated with care for the duration of hospital stay. ${ }^{7}$

Two reviewers independently extracted information on the ACE components, interventions, dose, and outcomes and entered it onto the data extraction form. One reviewer extracted and entered information on each component's objectives and approach, and another reviewer checked it. Disagreements on data extraction and entry were resolved by discussion and consensus, with assistance of a third team member when necessary. 


\section{Data Analysis}

For the first aim, descriptive statistics were used to identify the ACE components implemented as part of acute geriatric unit care. Information on the objectives, interventions, approach, and dose of the ACE components was analyzed using content analysis. Interventions applied in at least half of the trials are described in detail.

For the second aim, trial outcome data were used to calculate the mean ES associated with each ACE component. For continuous outcomes, the ES was calculated as the standardized difference in the means of the intervention and comparison groups using Cohen's formula. ${ }^{11}$ For dichotomous outcomes, odds ratios were calculated and then re-expressed as standardized mean differences using Chinn's formula. ${ }^{12}$ The mean ES associated with each ACE component was calculated for each individual outcome and for all of the outcomes combined. Cohen's benchmarks were used to interpret the magnitude of the ESs ( $<0.20$ (no effect), $0.20-0.49$ (small), 0.50 to 0.79 (medium), $\geq 0.80$ (large)). ${ }^{11}$

\section{RESULTS}

\section{Description of Trials and Papers}

Searches of all sources yielded 85,219 citations, of which 32 met the inclusion criteria (Figure 1). These included 19 articles, ${ }^{8,9,13-28}$ and one thesis ${ }^{29}$ reporting on 13 trials and 10 articles, 4-6, 30-36 $^{4}$ one abstract, ${ }^{37}$ and one manual, ${ }^{38}$ reporting supplemental ACE component information on the 13 trials. $^{8,}$ 9, 13-15, 17-19, 23, 26-29 One trial reported results related to the same intervention separately for two sites. ${ }^{15}$ These data were treated as two separate trials, resulting in14 included trials. Hence, the total number of 32 citations comprised 20 studies reporting on the effects of 14 trials and 12 articles reporting supplemental descriptive ACE component information. 
The 14 trials included a total of 6,839 participants. Descriptive information on participant and trial demographics, as well as outcomes reported in each trial, have been previously detailed. ${ }^{7}$

\section{Description of the ACE model components}

\section{Medical Review}

Medical review was reported in nine of the 14 trials. 8 , 9, 13, 14, 17, 23, 27-29 The objectives were to prevent iatrogenic complications $^{8,13,14,27-29}$ and functional deterioration ${ }^{8,9,17,29}$ associated with common hospital medications, treatments, and procedures. ${ }^{30}$ Medical review consisted of two main categories of interventions: assessment and delivery of interventions that addressed actual or potential problems identified in the assessment. Assessment involved screening for high risk medications $(n=8)$, treatments and planned procedures $(n=7)$. Results of the assessment determined the implementation of interventions or protocols to minimize the adverse effects of medications $(n=8)$, treatments, and planned procedures $(n=7)$ (Appendix 1, Table S1 of the electronic supplementary material). Where described, the protocols included standardized guidelines for dosing or avoiding medications, particularly psychoactive drugs ${ }^{4,8,9,17,27,30}$ and discontinuing at the earliest possible time or avoiding intravenous therapy, ${ }^{27,28}$ indwelling catheters, $^{\text {5, 9, 27-30 and physical restraints. }}{ }^{\text {4, 27, 28, } 30}$ Standardized guidelines were also used in performing venipuncture with small tubes, ${ }^{30}$ reviewing fluid and nutritional status administering bowel preparations or cathartics, ${ }^{30}$ prescribing intravenous fluids for those at risk of dehydration. $^{29,30}$; and prescribing intravenous fluids for those at risk of dehydration. ${ }^{27,30}$

With regard to dose, the medical review was most often initiated on admission $(n=4)$ and continued daily $(\mathrm{n}=8)$ for the duration of hospital stay $(\mathrm{n}=7)$. A mixed approach was used $(\mathrm{n}=$ 8) in which all medications, treatments, and planned procedures were screened and standardized guidelines implemented based on need. ${ }^{8,9,14,17,23, ~ 27-29 ~}$ 


\section{Early Rehabilitation}

Early rehabilitation was reported in seven of the 14 trials. ${ }^{8,9,13,14,17,28,29}$ The objectives were to prevent functional decline ${ }^{17,28,29}$ or to restore functional losses sustained during the acute phase of illness or injury. 5, 6, 8, 9, 14, 30, 32 Early rehabilitation consisted of assessing the need for physical therapy $(n=4)$ and providing physical $(n=6)$ and occupational $(n=5)$ therapy. Interventions focused most often on improving mobility to decrease the risk of falling $(n=6)$ and improving self-care ability $(n=4)$ by providing adaptive or assistive devices and exercises (both $n=4)$ (Appendix 1, Table S2 of the electronic supplementary material).

Dose of early rehabilitation was infrequently described. Where described, early rehabilitation was initiated within $24(n=1)$ to 72 hours of admission $(n=2)$ and performed daily $(n=1)$ for the duration of the hospital stay $(n=5)$. The approach to early rehabilitation varied according to the trials’ objectives. When the objective was to prevent functional decline, early rehabilitation was standardized and provided to all individuals $(\mathrm{n}=2)$ or mixed, in which at least a physical therapy assessment was provided $(n=1)$. In trials in which the objective was to restore functional losses, early rehabilitation was individualized and provided only to individuals who sustained functional losses $(n=3)$. In the latter approach, physical $(n=3)$ or occupational therapists $(n=2)$ participated in daily 30- to 60- minute interdisciplinary team meetings initiated within 24 hours of admission, during which information from daily patient-centered care assessments was communicated. ${ }^{31}$

\section{Early Discharge Planning}

Early discharge planning was reported in eight of the 14 trials. ${ }^{8,9,13-15,23,28}$ The objective was to facilitate transition of care to the community ${ }^{5,9,13,15,30}$ by maximizing the use of each hospital 
day $^{15}$ and facilitating healthcare provider, patient, and family or caregiver communication and agreement regarding the level of functional status needed to return home. ${ }^{5,13,15,30}$ Interventions included involving social workers and families or caregivers in care planning $(n=8)$, liaising

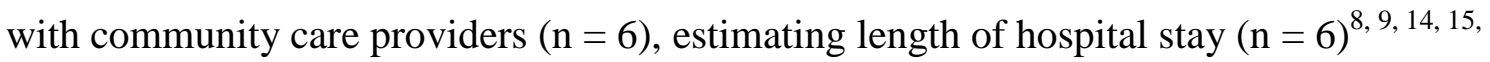
${ }^{23}$,developing a care plan outlining patient functional goals and home care needs, and developing strategies to meet functional goals and home care needs $(n=4)$. With regard to dose, early discharge planning was initiated on admission $(n=6)$ and continued daily $(n=5)$ for the duration of the hospital stay $(n=5)$. A standardized approach was used in which early discharge planning was provided to all patients and their families or caregivers $(n=4)$ (Appendix 1, Table S3 of the electronic supplementary material).

\section{Prepared Environment}

Prepared environment was reported in five of the 14 trials. $^{8,9,14,23,27}$ The objectives were to prevent cognitive $e^{8,9,14,23,27}$ and physical functional decline by fostering ambulation, 4, 32 functional independence, ${ }^{5,30}$ orientation, way-finding, familiarity, and socialization ${ }^{8,9,14,23}$ through physical environmental modifications. Environmental modifications involved the installation of clocks, calendars (both $n=5$ ), elevated toilet seats, easy-to-use door levers, corridor handrails, communal rooms (all $n=4$ ), designated spaces for personal items, carpeted flooring, visually contrasting floor and wall coverings, and enhanced lighting $($ all $n=3)$. Prepared environments were also those in which clutter was continually removed $(n=4)$. A standardized approach was used in which the modifications were applied to all intervention units (all five trials) (Appendix 1, Table S4 of the electronic supplementary material). Although the dose was not described, the environmental modifications were presumed permanent. 


\section{Patient-Centered Care}

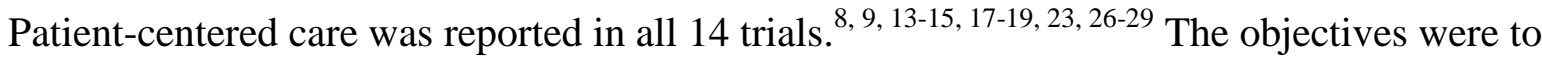
prevent functional decline ${ }^{5,6,13,14,17,26-30}$ and iatrogenic complications, ${ }^{28,31,32}$ and to preserve or restore functional status. ${ }^{5,6,14,30}$ Patient-centered care involved two categories of interventions: patient assessment and the delivery of interventions that addressed actual or potential problems identified in the assessment. Assessment most often comprised examinations of physical and cognitive status, specifically mobility, which included a falls risk assessment $(n=9)$ and cognition, which included confusion, delirium and delirium risk, or mental status assessments (n = 7) (Appendix 1, Table S5 of the electronic supplementary material). The interventions were focused on preserving or preventing decline in six main areas of functioning, or restoring states required for optimal functioning in those areas: mobility $(\mathrm{n}=11)$, hydration and nutritional status ( $n=10)$, cognition $(n=9)$, self-care ability $(n=9)$, continence or elimination $(n=8)$,, and skin integrity $(n=8)$.

With regard to dose, patient-centered care was initiated within 24 hours of admission (n = 7) and continued at least daily $(n=7)$ for the duration of the hospital stay $(n=10)$. A mixed approach was applied $(\mathrm{n}=8)$ that included standardized assessment, standardized implementation of preventative interventions for all patients, and individualized preventative or restorative interventions responsive to needs identified in the assessment. Standardized preventative interventions were "activated on all patients"5(p. 70) and were referred to in some papers as preventative protocols. $^{5,30}$

Standardized interventions focused on:

1. mobility (standing or ambulating at least 3 times a day ${ }^{30}$ or within 24 hours postoperatively $^{29}$ ); 
2. hydration and nutrition (providing nutritious snacks, ${ }^{5,}{ }^{29}$ high protein meals, ${ }^{29}$ and " 4 glasses of water a day - prescribed and administered like a drug” 27(p. 2031);

3. cognition (providing reminders of the day, date, and location; ensuring eye glasses and hearing aides were worn ${ }^{27}$; encouraging families to visit; administering routine pain medication to injured individuals ${ }^{29}$; employing the interventions identified above that focused on mobility, hydration and nutrition; and employing strategies to promote sleep, including avoiding treatments, providing warm drinks, ${ }^{27}$ and reducing noise ${ }^{29}$ at bedtime);

4. self-care ability (encouraging self-bathing ${ }^{5}$ );

5. skin-integrity (providing pressure-reducing mattresses). ${ }^{5}$

\section{Outcome Achievement Relative to ACE Components}

Medical review, early rehabilitation, and patient-centered care manifested the largest mean ESs (all ES $=0.20$ ) averaged across all outcomes of interest, followed by early discharge planning $(E S=0.17)$, and prepared environment $(E S=0.11)($ Table 1$)$. Medical review and early rehabilitation had the largest proportion (both 33\%) of ESs significant at the $\mathrm{p}<.05$ level, followed by patient-centered care (25\%), early discharge planning (16\%), and prepared environment (13\%).

Medical review and early rehabilitation had moderate ESs for falls and pressure ulcers and small ESs for delirium and functional decline. Patient-centered care had a moderate ES for pressure ulcers and small ESs for falls, delirium, and functional decline. Early discharge planning had small ESs for pressure ulcers and functional decline. Prepared environment had a small ES for delirium. 


\section{DISCUSSION}

The results of the ES analysis suggest that specific ACE component interventions of medical review, early rehabilitation, and patient-centered care appear to be optimal for overall outcome achievement and for reducing iatrogenic complications and functional decline for older adults admitted to hospital for an acute event.

\section{Implications for Practice and Policy}

Service providers who are unable to implement the ACE model in its entirety and wish to adapt the model to their existing contexts of practice may consider focusing their attention on medical review, early rehabilitation, and patient-centered care. These three ACE components and associated interventions may be adopted as evidence-based leading practices for acute hospital care of older adults (Table 2). With the increasing prevalence of chronic diseases in an aging population, service providers can anticipate that future hospital populations will be older and

have preexisting chronic health conditions. ${ }^{39}$ Multiple chronic health conditions tend to co-exist ${ }^{39}$ and may precipitate an acute event or complicate its management. Individuals with multiple chronic health conditions are particularly vulnerable to experiencing functional decline, ${ }^{40}$ and therefore require a function-focused approach to their care. Consequently, moving ACE practices into the "mainstream of hospital care" as envisioned by ACE pioneers ${ }^{38}$ will become even more critical to improving patient and system level outcomes.

\section{Limitations}

This study did not aim to compare isolated ACE components with usual care or to perform a meta-analysis that provides estimates of isolated component effects. Consequently, the average 
ES estimates represent the association between reported implementation of ACE components and the outcomes.

Because of the limited number of trials reporting on the outcomes of interest, inferential statistics could not be used to examine outcome differences in relation to implementation of ACE components. The number of trials included in the ES estimates on iatrogenic complications was small and may have resulted in an overestimation of their magnitude. ${ }^{11}$ ES differences among the five components are modest and may have been susceptible to bias in reporting in trials or by investigators. Also, one trial indicated that prepared environment had been implemented hospitalwide on usual care units midway through the trial. ${ }^{14}$ It is possible that easily implemented aspects of prepared environment, such as the installation of elevated toilets seats, clocks, and calendars, may have become standard features of usual care units in others trials,thus contributing to an underestimation of the ES of this ACE component.

\section{Implications for Research}

Future trials should examine the effects of isolated ACE components on outcomes. This would permit a future meta-analysis that provides estimates of each ACE component's effects, giving weight to the size of different trials included.

Future research should also examine the effectiveness of the combination of medical review, early rehabilitation, and patient-centered care. These three components share the objective of preventing functional decline either directly by focusing on improving mobility or indirectly by focusing on reducing hospital treatments, practices, or procedures that impede mobility. Researchers may use the findings that characterize ACE components to develop intervention protocols and train interventionists to enhance implementation fidelity, thereby increasing the internal validity of future effectiveness trials. 
Dose of early rehabilitation was infrequently described in the articles, which limits the ability to draw conclusions about its optimal dose for positive outcomes. With the narrow window of opportunity in which older adults' functional losses can be restored ${ }^{41}$ and serviceproviders’ need for direction on implementing early rehabilitation, future research should attend to describing its dose.

Early discharge planning had negligible ESs on discharge destination outcomes. It is possible that discharge destination outcomes do not capture the construct of transition of care, which was described as the objective of this ACE component. Future research should explore different measures of discharge destination and evaluate additional outcomes such as community provider and family or caregiver satisfaction with hospital communication and perception of the coordination and continuity of care across settings. ${ }^{42}$ In addition, future research should investigate how different healthcare professionals collaborate in implementing the ACE components to enhance outcome achievement. 


\section{ACKNOWLEDGMENTS}

The authors wish to thank research assistants Michael Vertolli, Mohamed Al-Haj, and Michelle How Pak Hing for their help with the literature search and decision-making partners Ms. Tiziana Rivera, Chief Practice Officer, York Central Hospital, and Dr. Mary Ferguson-Paré, former VicePresident of Professional Affairs and Chief Nurse Executive, University Health Network, for their advice on the grant which supported this study. Oral presentations based in part on the study findings were given at the annual conferences of the Canadian Association for Health Services and Policy Research May 30, 2012, and Canadian Association on Gerontology, October 20,2012.

Conflict of Interest: The editor in chief has reviewed the conflict of interest checklist provided by the authors and has determined that the authors have to financial or any kind of personal conflicts with this paper.

Financial support provided by the Canadian Institutes of Health Research (CIHR) Grant KRS-94307. Mary Fox was supported by an Ontario Ministry of Health and Long-Term Care Career Scientist Award, Kelly O'Brien by a CIHR Fellowship, Souraya Sidani and Dina Brooks by Canada Research Chairs, and Deborah Tregunno by an Ontario Ministry of Health and LongTerm Care Senior Nurse Research Award while conducting this study.

Author Contributions: Study concept: Fox. Study design: Fox, Sidani, Tregunno, Maimets, Brooks, O'Brien. Literature searching and initial records screening: Fox, Persaud, Maimets. Abstract and article screening for eligbility: Fox, Sidani, Persaud, Tregunno, Brooks, O'Brien. Data extraction: Fox, Sidani, Persaud, Tregunno, Brooks, O'Brien. Data analysis and manuscript preparation: Fox. Data interpretation: Fox, Sidani. Editing of manuscript: Sidani, Tregunno, Maimets, Brooks, O'Brien.

Sponsor's Role: None. 


\section{SUPPORTING INFORMATION}

Additional Supporting Information may be found in the online version of this article:

Appendix S1. Description of Reported ACE Components in each Trial.

Table S1. Medical Review.

Table S2. Early Rehabilition.

Table S3. Early Discharge Planning.

Table S4. Prepared Environment.

Table S5. Patient-Centered Care.

Please note: Wiley-Blackwell is not responsible for the content, accuracy, errors, or functionality of any supporting materials supplied by the authors. Any queries (other than missing material) should be directed to the corresponding author for the article. 


\section{GRAPHICS}

Table 1. Effects Sizes of Outcomes by ACE Component ${ }^{\mathrm{a}}$

\begin{tabular}{|c|c|c|c|c|c|c|c|c|c|c|}
\hline \multirow[t]{4}{*}{ Outcomes } & \multicolumn{10}{|c|}{ Components } \\
\hline & \multirow{2}{*}{\multicolumn{2}{|c|}{ Medical Review }} & \multicolumn{2}{|l|}{ Early } & \multicolumn{2}{|c|}{ Early Discharge } & \multicolumn{2}{|c|}{ Prepared } & \multicolumn{2}{|c|}{ Patient-centered } \\
\hline & & & Rehabilitati & & Plann & & Environ & & Care & \\
\hline & $\begin{array}{l}\text { ES, Mean } \\
\text { (Range) }\end{array}$ & $\mathbf{n} / \mathbf{N}$ & $\begin{array}{l}\text { ES, } \\
\text { Mean(Range) }\end{array}$ & $\mathbf{n} / \mathbf{N}$ & $\begin{array}{l}\text { ES, Mean } \\
\text { (Range) }\end{array}$ & $\mathbf{n} / \mathbf{N}$ & $\begin{array}{l}\text { ES, Mean } \\
\text { (Range) }\end{array}$ & $\mathbf{n} / \mathbf{N}$ & $\begin{array}{l}\text { ES, Mean } \\
\text { Range) }\end{array}$ & $\mathbf{n} / \mathbf{N}$ \\
\hline All Outcomes ${ }^{\mathrm{C}}$ & 0.20 & & 0.20 & & 0.17 & & 0.11 & & 0.20 & \\
\hline Falls & $\begin{array}{l}0.56 \\
(\mathrm{~N} / \mathrm{A})\end{array}$ & $1 / 1$ & $\begin{array}{l}0.56 \\
\text { (N/A) }\end{array}$ & $1 / 1$ & $\begin{array}{l}0.08(-.10- \\
0.25)\end{array}$ & $0 / 2$ & N/A & $0 / 0$ & $\begin{array}{l}0.24(-0.10- \\
0.56)\end{array}$ & $1 / 3$ \\
\hline Pressure Ulcers & $\begin{array}{l}0.59 \\
(\mathrm{~N} / \mathrm{A})\end{array}$ & $1 / 1$ & $\begin{array}{l}0.59 \\
(\mathrm{~N} / \mathrm{A})\end{array}$ & $1 / 1$ & $\begin{array}{l}0.49 \quad(0- \\
0.98)\end{array}$ & $0 / 2$ & N/A & $0 / 0$ & $\begin{array}{l}0.52 \quad(0- \\
0.98)\end{array}$ & $1 / 3$ \\
\hline Delirium & $\begin{array}{l}0.25(-0.05- \\
0.51)\end{array}$ & $2 / 3$ & $\begin{array}{l}0.23(-0.05- \\
0.51)\end{array}$ & $1 / 2$ & $\begin{array}{l}0.05 \\
(\mathrm{~N} / \mathrm{A})\end{array}$ & $0 / 1$ & $\begin{array}{l}0.29 \\
(\mathrm{~N} / \mathrm{A})\end{array}$ & $1 / 1$ & $\begin{array}{l}0.25(-0.05- \\
0.51)\end{array}$ & $2 / 3$ \\
\hline Functional Decline & $\begin{array}{l}0.22 \quad(0- \\
0.55)\end{array}$ & $2 / 6$ & $\begin{array}{l}0.23 \quad(0- \\
0.55)\end{array}$ & $2 / 5$ & $\begin{array}{l}0.20 \quad(0- \\
0.55)\end{array}$ & $1 / 4$ & $\begin{array}{l}0.10 \quad(0- \\
0.17)\end{array}$ & $0 / 4$ & $\begin{array}{l}0.22 \quad(0- \\
0.55)\end{array}$ & $2 / 6$ \\
\hline
\end{tabular}




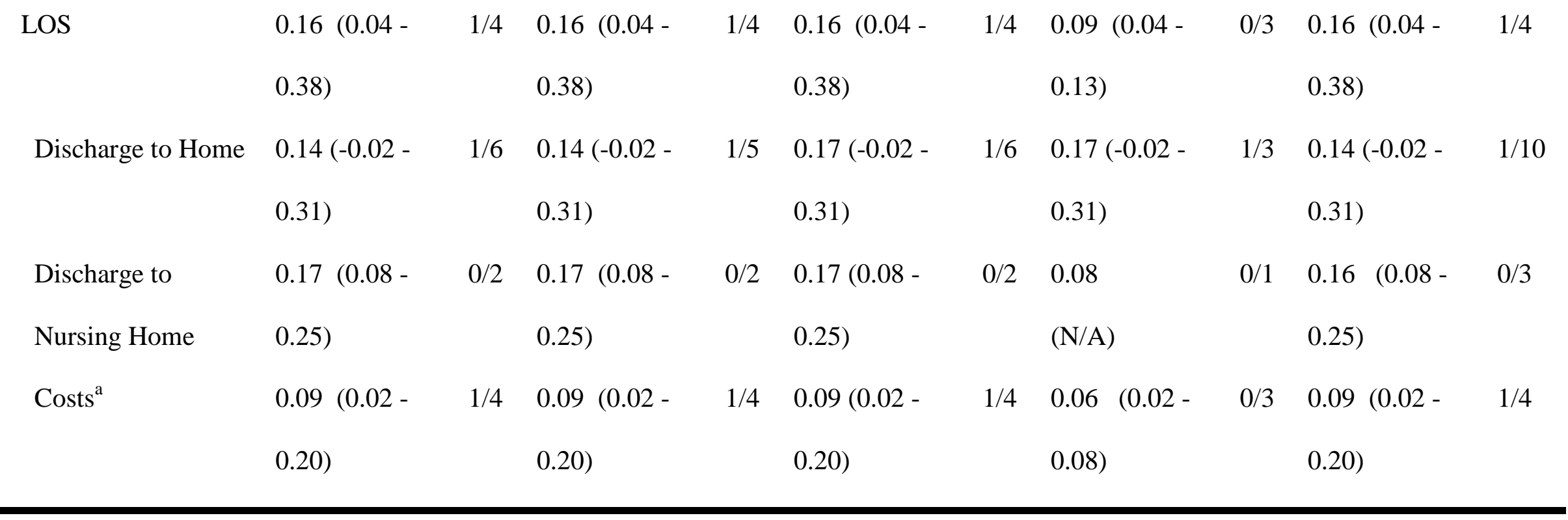

ES = effect size. LOS = length of hospital stay. $\mathrm{n} / \mathrm{N}=$ number of trials with significant ES/Total number of included trials. N/A = not applicable.

All ESs are standardized; only data from trials, and not from descriptive papers, were used in ES calculations. Raw data used in ES calculations can be found in Appendix 1, Table S3 of the online supporting information of a prior study. ${ }^{7}$

The direction of all effects was standardized so that all positive effects are in favor of the intervention group that received the ACE components.

$\mathrm{n} / \mathrm{N}=$ number of trials with significant ES/total number of included trials; N/A= not applicable. 
${ }^{\text {a }}$ Similar results were obtained when trials contributing to heterogeneity were included, with the exception of patient-centered care and costs, for which mean ES was 0.21 ( range $=0.02-.067 ; \mathrm{n}=2 / 5$ ). 
Table 2. Recommendations for Implementing Medical Review, Early Rehabilitation, and Patient-Centered Care

\section{Acute Care for Elders Component}

Medical review: Standardized admission

assessment and daily review of high risk

procedures for duration of hospital stay. medications, treatments and planned

\section{Recommendations}

Based on the assessment indicating need, standardized protocols should be implemented to:

Avoid or dose high risk medications, particularly psychoactive drugs

1. Avoid or discontinue at the earliest possible time intravenous therapy, indwelling catheters and physical restraints

2. Perform venipuncture with small tubes

3. Review fluid and nutritional status prior to administering bowel preparations or cathartics

4. Prescribe fluid and nutritional supplements for patients at risk of malnutrition and dehydration.

Early Rehabilitation: Assessment of the need for physical therapy. In hospitals where
Interventions should focus on improving:

Mobility through provision of assistive devices and exercises 
standard rehabilitation assessment is not

feasible, therapists should attend daily

interdisciplinary rounds where they can be

informed of older adults’ functional status

and need for rehabilitation. ${ }^{\text {a }}$

Patient-Centered Care: Standardized

assessment of physical and cognitive

functioning (focused on mobility, falls risk,

and delirium risk) within 24 hours of

admission and daily for duration of hospital

stay.
1. Self-care ability through provision of adaptive devices.
Based on assessment indicating need, individualized interventions should be provided to preserve or restore:

Mobility

1. Hydration and nutritional status

2. Cognition

3. Self-care ability

4. Continence/elimination

5. Skin integrity.

Regardless of assessment, the following should be standard preventative interventions: 
Stand or ambulate $>3$ times per day and within 24 hours postoperatively

- Provide nutritious snacks, high- protein meals, and four glasses of water per day

- Encourage self-bathing. Provide pressure-reducing mattresses

- Provide reminders of the day, date, and location

- Ensure eye glasses and hearing aides are worn. Foster family visits

- Administer routine pain medication to injured patients

- $\quad$ Reduce nighttime noise

- Offer warm drinks at bedtime.

${ }^{a}$ Because dose of early rehabilitation was infrequently described in the articles, it is not possible to recommend an optimal dose for positive outcomes. 


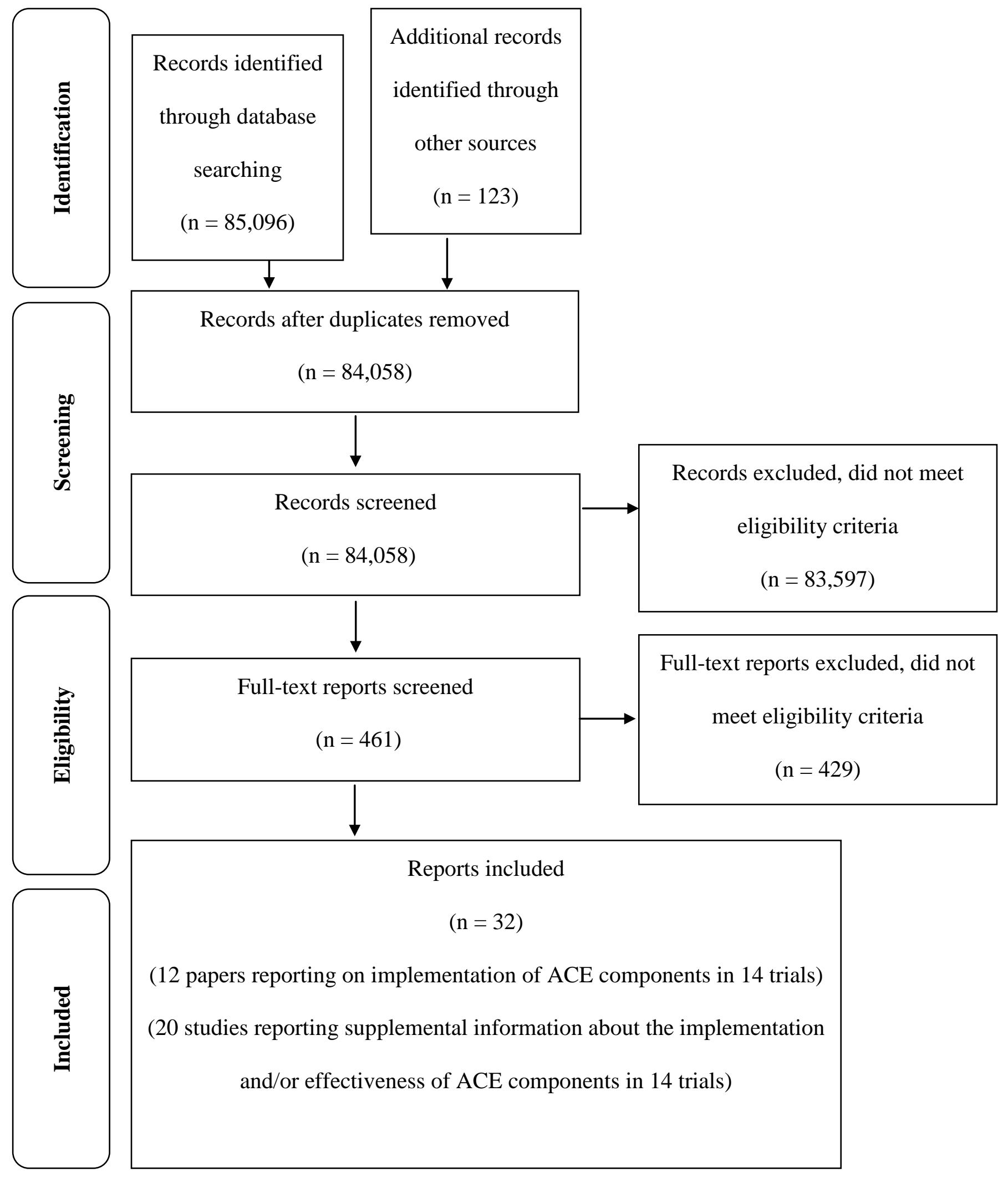

Figure 1. PRISMA Flow Diagram. ${ }^{43} \mathrm{ACE}=$ Acute Care for Elders. 


\section{APPENDIX S1: Description of Reported ACE Components in each Trial}

Table S1. Medical Review

\begin{tabular}{|c|c|c|c|c|c|c|c|c|c|c|c|c|c|}
\hline$\frac{\bar{E}}{\frac{\mathrm{g}}{2}}$ & $\stackrel{\mathscr{Z}}{\mathscr{E}}$ & 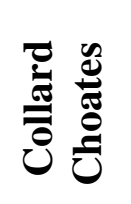 & 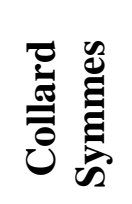 & 咆 & 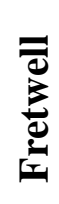 & 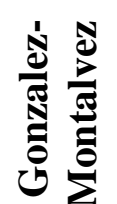 & 胥 & 窇 & 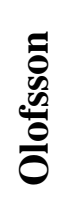 & 节 & 党 & 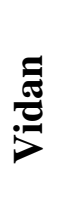 & $\frac{\pi}{\frac{\pi}{\pi}}$ \\
\hline
\end{tabular}

Interventions

Assessment of

Medications

Treatments $^{\mathrm{a}}$

Minimization of

High risk meds ${ }^{b}$

Treatments $^{\mathrm{a}}$

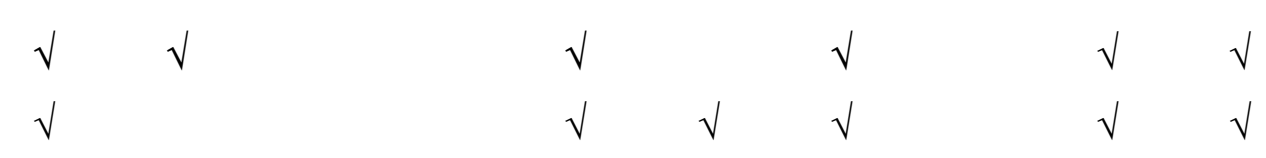

Initiation from adm NR NR

On adm

Within 24 hrs

Within 72 hrs

Frequency

QD

3X/wk

Duration

LOS 
LOS \& $2 \mathrm{mo}^{\mathrm{c}}$

LOS \& $4 \mathrm{mo}^{\mathrm{d}}$ $\sqrt{ }$

\section{Approach}

Mixed

NR

$\sqrt{ }$

$\sqrt{ }$

Adm = admission to hospital or unit, meds = medications, mo = month, hrs $=$ hours, LOS $=$ length of hospital stay, NR $=$ not reported,

$\mathrm{QD}=$ every day, wk = week, $\mathrm{X}=$ times.

${ }^{\mathrm{a}}$ Treatments and/or planned procedures.

${ }^{\mathrm{b}}$ High risk medications including polypharmacy.

${ }^{\mathrm{c}}$ One follow-up telephone call every week for one month followed by one telephone call at 2 months post-discharge.

${ }^{\mathrm{d}}$ One follow-up visit at 4 months post-discharge. 


\begin{tabular}{|c|c|c|c|c|c|c|c|c|c|c|c|c|c|c|}
\hline & $\frac{\bar{\Xi}}{\frac{\mathrm{B}}{2}}$ & 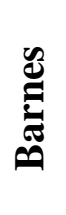 & 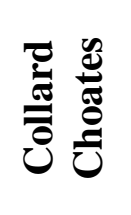 & 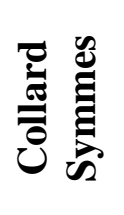 & 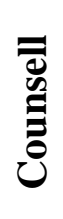 & 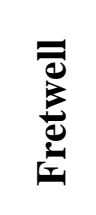 & 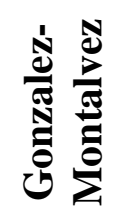 & 氠 & 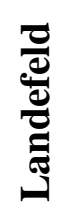 & 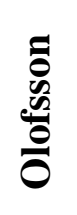 & 圐 & ڤ & $\underset{7}{\Xi}$ & $\frac{\pi}{\frac{\pi}{0}}$ \\
\hline \multicolumn{15}{|c|}{ Interventions } \\
\hline \multicolumn{15}{|l|}{ Assessment } \\
\hline Admission OT & $\sqrt{ }$ & & & & & & & & & $\sqrt{ }$ & & & & $\sqrt{ }$ \\
\hline Admission PT & $\sqrt{ }$ & & & & & $\sqrt{ }$ & & & & $\sqrt{ }$ & & & & $\sqrt{ }$ \\
\hline \multicolumn{15}{|l|}{ Intervention focus } \\
\hline OT treatment & $\sqrt{ }$ & & & & $\sqrt{ }$ & & & & $\sqrt{ }$ & $\sqrt{ }$ & & & & $\sqrt{ }$ \\
\hline PT treatment & $\sqrt{ }$ & $\sqrt{ }$ & & & $\sqrt{ }$ & $\mathrm{NR}^{\mathrm{a}}$ & & & $\sqrt{ }$ & $\sqrt{ }$ & & & & $\sqrt{ }$ \\
\hline ADL/self-care & & & & & $\sqrt{ }$ & & & & $\sqrt{ }$ & $\sqrt{ }$ & & & & $\sqrt{ }$ \\
\hline Assistive device provision & & $\sqrt{ }$ & & & $\sqrt{ }$ & & & & $\sqrt{ }$ & $\sqrt{ }$ & & & & \\
\hline Consultation $^{\mathrm{b}}$ & & & & & & & & & & $\sqrt{ }$ & & & & \\
\hline Coordination & & & & & & & & & $\sqrt{ }$ & & & & & \\
\hline Endurance & & & & & & & & & $\sqrt{ }$ & & & & & \\
\hline Exercises & & $\sqrt{ }$ & & & $\sqrt{ }$ & & & & $\sqrt{ }$ & $\sqrt{ }$ & & & & \\
\hline Mobility/falls prevention & $\sqrt{ }$ & $\sqrt{ }$ & & & $\sqrt{ }$ & $\sqrt{ }$ & & & $\sqrt{ }$ & $\sqrt{ }$ & & & & \\
\hline Neurosensory stimulation & & & & & & & & & & & & & & $\sqrt{ }$ \\
\hline Pain relief & & & & & & & & & $\sqrt{ }$ & & & & & \\
\hline Patient/family education & & & & & & & & & $\sqrt{ }$ & $\sqrt{ }$ & & & & $\sqrt{ }$ \\
\hline Range of motion & & & & & $\sqrt{ }$ & & & & $\sqrt{ }$ & & & & & \\
\hline
\end{tabular}




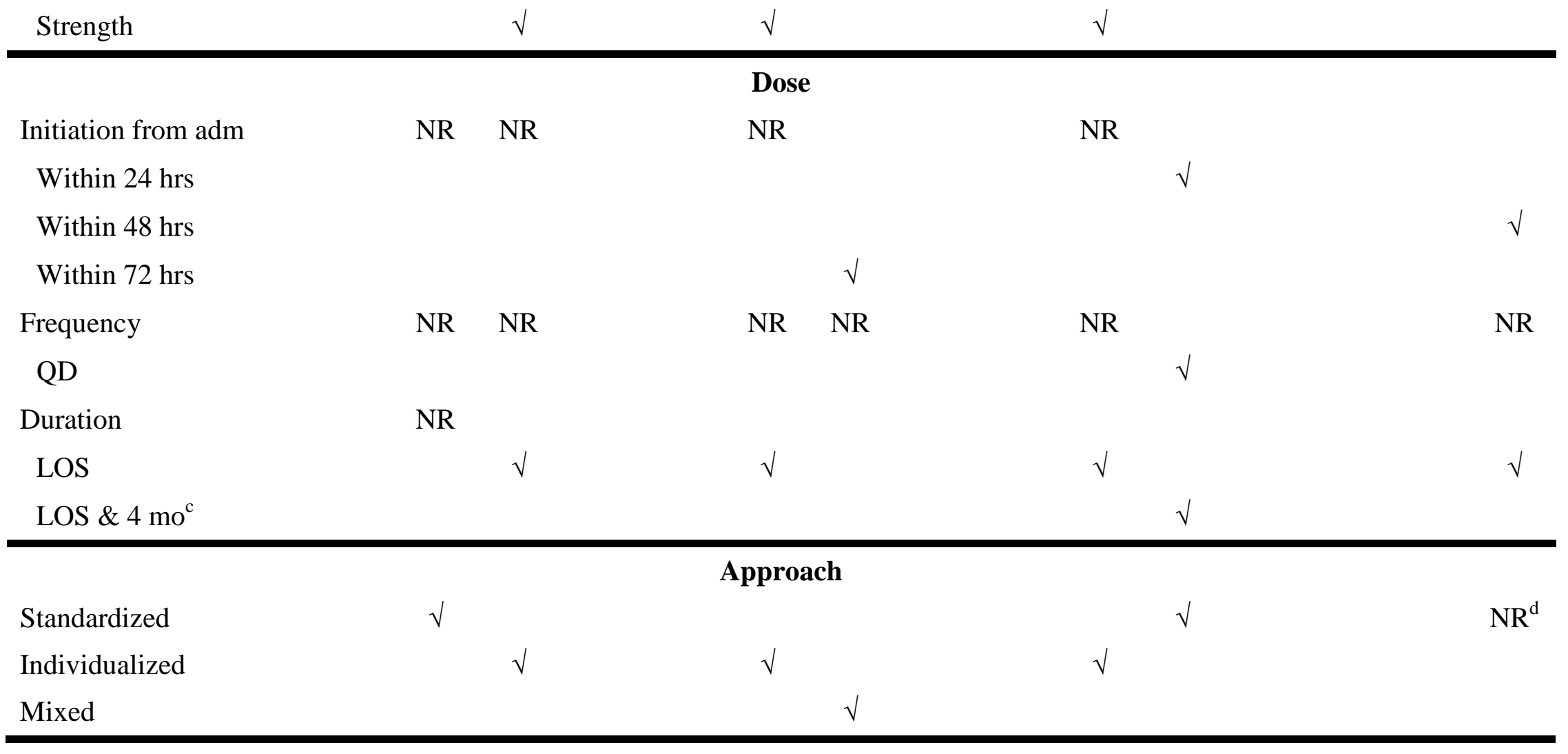

$\mathrm{ADL}=$ activities of daily living, adm = admission to hospital or unit, hrs = hours, LOS = length of hospital stay, mo $=$ month, $\mathrm{NR}=$ not reported, OT = occupational therapy, PT = physical therapy, QD = every day.

${ }^{a}$ Assessment performed and individualized care plan developed and shared with care staff. Unclear if physical therapy, other than assessment, provided.

${ }^{\mathrm{b}}$ Consultation with community providers. 
${ }^{\mathrm{c}}$ One follow-up phone call 2 weeks post-discharge and one follow-up home visit 4 months post-operatively.

${ }^{\mathrm{d}}$ All patients presumed. 
Table S3. Early Discharge Planning

\begin{tabular}{|c|c|c|c|c|c|c|c|c|c|c|c|c|c|}
\hline$\frac{\text { 夰 }}{\frac{\bar{z}}{4}}$ & 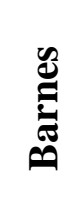 & 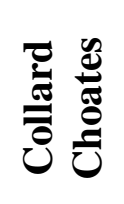 & 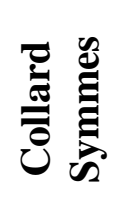 & 氖 & 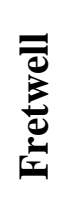 & 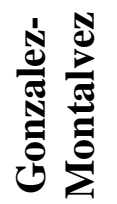 & 产 & 西 & $\begin{array}{l}\tilde{0} \\
\mathscr{0} \\
\frac{0}{0} \\
0\end{array}$ & 兽 & 㫕 & 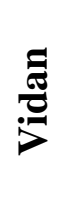 & $\frac{\pi}{\frac{\pi}{0}}$ \\
\hline
\end{tabular}

\section{Interventions}

Assessment of

Plans \& needs for discharge $\mathrm{e}^{\mathrm{a}}$

Home assessment

Intervention focus

Care plan development ${ }^{\mathrm{b}}$

Community provider liaison

Family involvement ${ }^{\mathrm{C}}$

Home environment

modification

LOS estimation

Social worker involvement

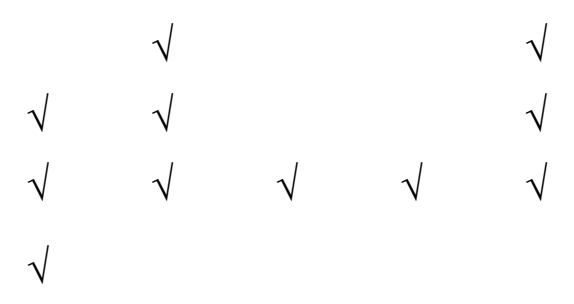

$\begin{array}{ll}\sqrt{ } & \sqrt{ } \\ \sqrt{ } & \sqrt{ } \\ \sqrt{ } & \sqrt{ }\end{array}$

Initiation from admission

On adm

Very early after adm

Within 48 hrs

Frequency

NR

NR NR

QD

$\begin{array}{lllll} & \sqrt{ } & \sqrt{ } & \sqrt{ } & \sqrt{ } \\ \sqrt{ } & \sqrt{ } & \sqrt{ } & \sqrt{ } & \sqrt{ }\end{array}$

\section{Dose}

$\sqrt{ }$

$\sqrt{ }$

$\checkmark$ 


\begin{tabular}{|c|c|c|c|c|c|c|c|c|}
\hline Duration & NR & & & & & & & \\
\hline LOS & & $\sqrt{ }$ & & & $\sqrt{ }$ & $\sqrt{ }$ & $\sqrt{ }$ & $\sqrt{ }$ \\
\hline LOS \& $3 w k s^{d}$ & & & $\sqrt{ }$ & $\sqrt{ }$ & & & & \\
\hline Standardized & NR & $\sqrt{ }$ & NR & NR & $\sqrt{ }$ & $\sqrt{ }$ & $\sqrt{ }$ & NR \\
\hline
\end{tabular}

$\mathrm{ADL}=$ activities of daily living, adm = admission to hospital or unit, hrs = hours, LOS = length of hospital stay, NR = not reported, QD = every day, wks = weeks.

${ }^{\mathrm{a}}$ With emphasis on goal of returning home.

${ }^{\mathrm{b}}$ Care plan development outlining goals, functional status required to return home, and strategies to achieve goals.

${ }^{\mathrm{c}}$ Includes involving family in patient care and/or care planning, providing family education, and/ or conducting family conference.

${ }^{\mathrm{d}}$ One follow-up visit at 3 weeks post-discharge. 


\begin{tabular}{|c|c|c|c|c|c|c|c|c|c|c|c|c|c|c|}
\hline & $\frac{\bar{\Xi}}{\mathrm{E}}$ & $\underset{\mathscr{E}}{\mathscr{E}}$ & 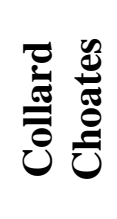 & 囦 & 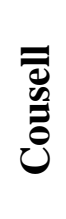 & 胥 & 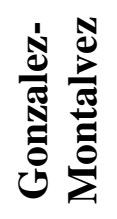 & 疍 & 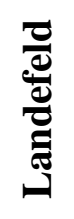 & 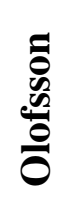 & 莽 & 苞 & $\underset{j}{\text { 坖 }}$ & $\frac{\pi}{\widetilde{J}}$ \\
\hline \multicolumn{15}{|c|}{ Interventions } \\
\hline \multicolumn{15}{|l|}{ Installation/construction of } \\
\hline Clocks \& calendars & & $\sqrt{ }$ & & & $\sqrt{ }$ & & & & $\sqrt{ }$ & & $\sqrt{ }$ & & $\sqrt{ }$ & \\
\hline Communal dining room ${ }^{\mathrm{a}}$ & & $\sqrt{ }$ & & & $\sqrt{ }$ & & & & $\sqrt{ }$ & & $\sqrt{ }$ & & & \\
\hline Easy to use door levers & & $\sqrt{ }$ & & & $\sqrt{ }$ & & & & $\sqrt{ }$ & & $\sqrt{ }$ & & & \\
\hline Elevated toilet seats & & $\sqrt{ }$ & & & $\sqrt{ }$ & & & & $\sqrt{ }$ & & $\sqrt{ }$ & & & \\
\hline Floor lighting & & $\sqrt{ }$ & & & $\sqrt{ }$ & & & & $\sqrt{ }$ & & & & & \\
\hline Handrails in corridors & & $\sqrt{ }$ & & & $\sqrt{ }$ & & & & $\sqrt{ }$ & & $\sqrt{ }$ & & & \\
\hline Lighting behind beds & & $\sqrt{ }$ & & & $\sqrt{ }$ & & & & $\sqrt{ }$ & & & & & \\
\hline Night lights & & $\sqrt{ }$ & & & & & & & & & & & & \\
\hline Non-slip flooring & & $\sqrt{ }$ & & & $\sqrt{ }$ & & & & $\sqrt{ }$ & & & & & \\
\hline Padded hallway seats & & $\sqrt{ }$ & & & & & & & & & & & & \\
\hline Space for personal items & & $\sqrt{ }$ & & & $\sqrt{ }$ & & & & $\sqrt{ }$ & & & & & \\
\hline $\begin{array}{l}\text { Visually contrasting carpets \& } \\
\text { wall coverings }\end{array}$ & & $\sqrt{ }$ & & & $\sqrt{ }$ & & & & $\sqrt{ }$ & & & & & \\
\hline Wall finish/paint ${ }^{\mathrm{b}}$ & & $\sqrt{ }$ & & & $\sqrt{ }$ & & & & $\sqrt{ }$ & & & & & \\
\hline Removal of clutter/hazards ${ }^{c}$ & & $\sqrt{ }$ & & & $\sqrt{ }$ & & & & $\sqrt{ }$ & & $\sqrt{ }$ & & & \\
\hline \multicolumn{15}{|c|}{ Dose $^{d}$} \\
\hline
\end{tabular}




\begin{tabular}{lcccc}
\hline & Approach & & \\
Standardized & $\sqrt{ }$ & $\sqrt{ }$ & $\sqrt{ }$ & $\sqrt{ }$ \\
\hline
\end{tabular}

${ }^{a}$ For patient and family/caregiver use.

${ }^{\mathrm{b}}$ Non-glare/appealing wall finish/paint applied.

${ }^{\mathrm{c}}$ Continual removal of environmental clutter and hazards in rooms and hallways.

${ }^{\mathrm{d}}$ Dose was not described, but it was presumed to be permanent. 


Table S5. Patient-Centered Care

Interventions

Assessment of

\begin{tabular}{|c|c|}
\hline ADL/self-care & $\sqrt{ }$ \\
\hline Cognition $^{\mathrm{a}}$ & \\
\hline Continence/elimination & $\sqrt{ }$ \\
\hline Educational needs ${ }^{\mathrm{b}}$ & \\
\hline Hydration/nutrition & \\
\hline Mobility/falls & $\sqrt{ }$ \\
\hline Mood $^{\mathrm{c}}$ & \\
\hline Nutrition & \\
\hline Pain & $\sqrt{ }$ \\
\hline Skin integrity & \\
\hline Sleep & \\
\hline Other $^{\mathrm{d}}$ & \\
\hline
\end{tabular}

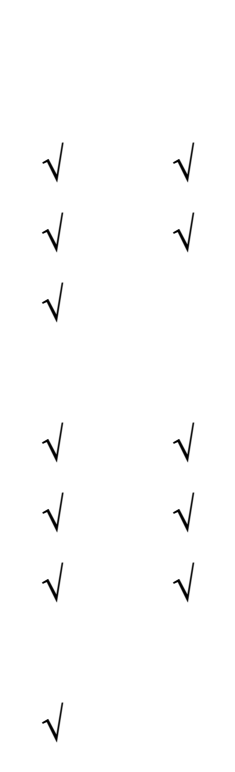

Intervention focus

$\begin{array}{lcccccc}\text { ADL/self-care } & \sqrt{ } & \sqrt{ } & \sqrt{ } & \sqrt{ } & \sqrt{ } & \\ \text { Cognition }^{\text {a }} & \sqrt{ } & \sqrt{ } & & & \sqrt{ } & \sqrt{ } \\ \text { Continence/elimination } & \sqrt{ } & \sqrt{ } & & & \sqrt{ } & \sqrt{ }\end{array}$




\begin{tabular}{|c|c|c|c|c|c|c|c|c|c|c|c|c|c|c|}
\hline Goal identification & $\sqrt{ }$ & & & & $\sqrt{ }$ & & & & $\sqrt{ }$ & $\sqrt{ }$ & $\sqrt{ }$ & & & \\
\hline Hearing \& vision & $\sqrt{ }$ & & & & $\sqrt{ }$ & & & & $\sqrt{ }$ & & $\sqrt{ }$ & & $\sqrt{ }$ & \\
\hline Hydration/nutrition & $\sqrt{ }$ & $\sqrt{ }$ & $\sqrt{ }$ & $\sqrt{ }$ & $\sqrt{ }$ & $\sqrt{ }$ & & & $\sqrt{ }$ & $\sqrt{ }$ & $\sqrt{ }$ & & $\sqrt{ }$ & \\
\hline Mobility/falls & $\sqrt{ }$ & $\sqrt{ }$ & $\sqrt{ }$ & $\sqrt{ }$ & $\sqrt{ }$ & $\sqrt{ }$ & $\sqrt{ }$ & & $\sqrt{ }$ & $\sqrt{ }$ & $\sqrt{ }$ & & $\sqrt{ }$ & \\
\hline $\operatorname{Mood}^{\mathrm{c}}$ & $\sqrt{ }$ & $\sqrt{ }$ & & & $\sqrt{ }$ & $\sqrt{ }$ & & & $\sqrt{ }$ & & $\sqrt{ }$ & & & \\
\hline Oral hygiene & & & & & $\sqrt{ }$ & & & & & $\sqrt{ }$ & & & & \\
\hline Pain & $\sqrt{ }$ & & & & & & & & & $\sqrt{ }$ & & & & \\
\hline $\begin{array}{l}\text { Patient/family } \\
\text { education }\end{array}$ & & & & & $\sqrt{ }$ & & & & $\sqrt{ }$ & $\sqrt{ }$ & $\sqrt{ }$ & & & $\sqrt{ }$ \\
\hline Skin integrity & $\sqrt{ }$ & $\sqrt{ }$ & & & $\sqrt{ }$ & & & & $\sqrt{ }$ & $\sqrt{ }$ & $\sqrt{ }$ & & $\sqrt{ }$ & $\sqrt{ }$ \\
\hline Sleep & $\sqrt{ }$ & & & & $\sqrt{ }$ & & & & $\sqrt{ }$ & $\sqrt{ }$ & $\sqrt{ }$ & & $\sqrt{ }$ & \\
\hline \multicolumn{15}{|c|}{ Dose } \\
\hline Initiation from adm & NR & & & & & & & NR & & & & & & \\
\hline Pre-adm & & & $\sqrt{ }$ & $\sqrt{ }$ & & & & & & & & & & \\
\hline On adm & & & $\sqrt{ }$ & $\sqrt{ }$ & & & $\sqrt{ }$ & & & & & & & \\
\hline Within 24 hrs & & $\sqrt{ }$ & & & $\sqrt{ }$ & $\sqrt{ }$ & & & $\sqrt{ }$ & $\sqrt{ }$ & $\sqrt{ }$ & & $\sqrt{ }$ & \\
\hline Within 48 hrs & & & & & & & & & & & & & & $\sqrt{ }$ \\
\hline Within 72 hrs & & & & & & & & & & & & $\sqrt{ }$ & & \\
\hline Frequency & NR & & NR & NR & & NR & NR & NR & & & & NR & & \\
\hline QD & & $\sqrt{ }$ & & & $\sqrt{ }$ & & & & & & & & $\sqrt{ }$ & $\sqrt{ }$ \\
\hline QD to TID & & & & & & & & & $\sqrt{ }$ & $\sqrt{ }$ & $\sqrt{ }$ & & & \\
\hline Duration & & & & & & & NR & & & & & NR & & \\
\hline LOS & $\sqrt{ }$ & $\sqrt{ }$ & $\sqrt{ }$ & $\sqrt{ }$ & $\sqrt{ }$ & & & $\sqrt{ }$ & $\sqrt{ }$ & & $\sqrt{ }$ & & $\sqrt{ }$ & $v$ \\
\hline
\end{tabular}


LOS \& $2 \mathrm{mo}^{\mathrm{e}}$

LOS \& $4 \mathrm{mo}^{\mathrm{f}}$ $\sqrt{ }$

Approach

NR NR V

Standardized

Individualized

Mixed

$\sqrt{ }$

$\sqrt{ }$

$\mathrm{ADL}=$ activities of daily living, adm $=$ admission to hospital or unit, hrs $=$ hours, LOS $=$ length of hospital stay, $\mathrm{mo}=$ month, $\mathrm{NR}=$ not reported, QD = every day, TID = 3 times a day.

${ }^{a}$ Cognition includes confusion, mental functioning, and/or delirium.

${ }^{\mathrm{b}}$ Educational needs of patient and/or family/caregivers.

${ }^{\mathrm{c}}$ Mood includes emotion, anxiety, and/or depression.

${ }^{\mathrm{d}}$ Other includes geriatric, generalized, or comprehensive assessment not specified.

${ }^{\mathrm{e}}$ Five follow-up telephone calls every week for one month then one follow-up call 2 months after hospital discharge.

${ }^{\mathrm{f}}$ One follow-up visit 4 months post-operatively. 


\section{REFERENCES}

1. Walsh KA, Bruza JM. Review: Hospitalization of the elderly. Ann Long Term Care 2007;15:18-23.

2. Sager MA, Franke T, Inouye SK et al. Functional outcomes of acute medical illness and hospitalization in older persons. Arch Intern Med 1996;156:645-652.

3. Covinsky KE, Palmer RM, Fortinsky RH et al. Loss of independence in activities of daily living in older adults hospitalized with medical illnesses: Increased vulnerability with age. J Am Geriatr Soc 2003;51:451-458.

4. $\quad$ Palmer RM, Counsell SR, Landefeld SC. Acute care for elders units: Practical considerations for optimizing health outcomes. Dis Manag Health Outcomes 2003;11:507-517. 5. Covinsky KE, Palmer RM, Kresevic DM et al. Improving functional outcomes in older patients: Lessons from an acute care for elders unit. Jt Comm J Qual Improv 1998;24:63-76.

6. Palmer RM, Counsell S, Landefeld SS. Clinical intervention trials: The ACE unit. Clin Geriatr Med 1998;14:831-849.

7. Fox MT, Persaud M, Maimets I et al. Effectiveness of acute geriatric unit care using Acute Care for Elders components: A systematic review and meta-analysis. J. Am Geriatr Soc 2012;60:2237-2245.

8. Counsell SR, Holder CM, Liebenauer LL et al. Effects of a multicomponent intervention on functional outcomes and process of care of hospitalized older patients: A randomized controlled trial of Acute Care for Elders (ACE) in a community hospital. J Am Geriatr Soc 2000;48:1572-1581.

9. Landefeld CS, Palmer RM, Kresevic DM et al. A randomized trial of care in a hospital medical unit especially designed to improve the functional outcomes of acutely ill older patients. N Engl J Med 1995;332:1338-1334.

10. Wong RYM, Shaw M, Acton $\mathrm{C}$ et al. An interdisciplinary approach to optimize health services in a specialized Acute Care for Elders unit. Geriatr Today 2003;6:177-186.

11. Grissom RJ, Kim JJ. Effect Sizes for Research: Univariate and Multivariate Applications, 2nd Ed. New York: Routledge, 2012.

12. Chinn S. A simple method for converting an odds ratio to effect size for use in metaanalysis. Stat Med 2000;19:3127-3131.

13. Asplund K, Gustafson Y, Jacobsson C et al. Geriatric-based versus general wards for older acute medical patients: A randomized comparison of outcomes and use of resources. J Am Geriatr Soc 2000;48:1381-1388.

14. Barnes DE, Palmer RM, Kresevic DM et al. Acute Care For Elders units produced shorter hospital stays at lower cost while maintaining patients' functional status. Health Aff 2012;31:1227-1236.

15. Collard AF, Bachman SS, Beatrice DF. Acute care delivery for the geriatric patient: An innovative approach. QRB Qual Rev Bull 1985;11:180-5.

16. Covinsky KE, King. JT, Quinn LM et al. Do Acute Care for Elders units increase hospital costs? A cost analysis using the hospital perspective. J Am Geriatr Soc 1997;45:729-734.

17. Fretwell MD, Raymond PM, McGarvey ST et al. The Senior Care Study. A controlled trial of a consultative/unit-based geriatric assessment program in acute care. J Am Geriatr Soc 1990;38:1073-1081.

18. Gonzalez-Montalvo JI, Alarcon T, Mauleon JL et al. The orthogeriatric unit for acute patients: A new model of care that improves efficiency in the management of patients with hip fracture. Hip Int 2010;20:229-235. 
19. Harris RD, Henschke PJ, Popplewell PY et al. A randomised study of outcomes in a defined group of acutely ill elderly patients managed in a geriatric assessment unit or a general medical unit. Aust N Z J Med 1991;21:230-234.

20. Lundstrom M, Olofsson B, Stenvall $\mathrm{M}$ et al. Postoperative delirium in old patients with femoral neck fracture: A randomized intervention study. Aging Clin Exp Res 2007;19:178-186. 21. Olofsson B, Stenvall M, Lundstrom $M$ et al. Malnutrition in hip fracture patients: An intervention study. J Clin Nurs 2007;16:2027-2038.

22. Owens NJ, Sherburne NJ, Silliman RA et al. The Senior Care Study. The optimal use of medications in acutely ill older patients. J Am Geriatr Soc 1990;38:1082-1087.

23. Somme D, Andrieux N, Guerot E et al. Loss of autonomy among elderly patients after a stay in a medical intensive care unit (ICU): A randomized study of the benefit of transfer to a geriatric ward. Arch Gerontol Geriatrics 2010;50:e36-e40.

24. Stenvall M, Ologsson B, Nyberg L et al. Improved performance in activities of daily living and mobility after a multidisciplinary postoperative rehabilitation in older people with femoral neck fracture: A randomized controlled trial with 1-year follow up. J Rehabil Med 2007;39:232-238.

25. Stenvall M, Ologsson B, Lundstrom $\mathrm{M}$ et al. A multidisciplinary, multifactorial intervention program reduces postoperative falls and injuries after neck fracture. Osteoporos Int 2007;18:167-175.

26. Stewart M, Suchak N, Scheve A et al. The impact of a geriatrics evaluation and management unit compared to standard care in a community teaching hospital. Md Med J 1999;48:62-67.

27. Vidan MT, Sanchez E, Alonso M et al. An intervention integrated into daily clinical practice reduces the incidence of delirium during hospitalization in elderly patients. J Am Geriatr Soc 2009;57:2029-2036.

28. Zelada MA, Salinas R, Baztan J. Reduction of functional deterioration during hospitalization in an acute geriatric unit. Arch Gerontol Geriatr 2009;48:35-39.

29. Olofsson B. Old People with Femoral Neck Fracture: Delirium, Malnutrition and Surgical Methods_-an Intervention Program [dissertation]. Umea: Umea University, 2007.

30. Palmer RM, Landefeld CS, Kresevic DM et al. A medical unit for the acute care of the elderly. J Am Geriatr Soc 1994;42:545-552.

31. Kresevic D, Holder C. Interdisciplinary care. Clin Geriatr Med 1998;14:788-798.

32. Kresevic DM, Counsell SR, Covinsky K et al. A patient-centered model of acute care for elders. Nurs Clin North Am 1998;33:515-527.

33. "Prehab program" can slow down functional decline in geriatric patients. Patient Focus Care Satisf 1998;6:17-28.

34. Sletvold O, Tilvis R, Jonsson A et al. Geriatric work-up in the Nordic countries. Dan Med Bull 1996;43:350-359.

35. Bachman SS, Collard AF, Greenberg JN et al. An innovative approach to geriatric acute care delivery: The Choate-Symmes experience. Hosp Health Serv Admin 1987;32:509-520.

36. Jónsson A, Gustafson Y, Schroll M et al. Geriatric rehabilitation as an integral part of geriatric medicine in the Nordic countries. Dan Med Bull 2003;50:439-445.

37. Palmer RM, Landefeld CS, Kresevic D et al. A medical unit for the acute care of hospitalized elderly patients: Conceptual basis and feasibility. J Am Geriatr Soc 1991;39:A62. 38. Counsell SR, Holder C, Liebenauer LL et al. The Acute Care for Elders (ACE) Manual: Meeting the Challenge of Providing Quality and Cost-Effective Hospital Care to Older Adults. Akron, OH: Summa Health System, 1998. 
39. Haydon E, Roerecke M, Giesbrecht N et al. Chronic Disease in Ontario and Canada: Determinants, Risk Factors and Prevention Priorities: Report Prepared for the Ontario Chronic Disease Prevention Alliance and the Ontario Public Health Association; 2006 [on-line]. Available at http://www.ocdpa.on.ca/OCDPA/docs/CDP-FullReport-Mar06.pdf Accessed August 15, 2012. 40. Wolff JL, Starfield B, Anderson G. Prevalence, expenditures, and complications of multiple chronic conditions in the elderly. Arch Intern Med 2002;162:2269-2276.

41. Boyd CM, Landefeld S, Counsell SR et al. Recovery in activities of daily living among older adults following hospitalization for acute medical illness. J Am Geriatr Soc 2008;56:21712179.

42. NTOCC Measures Work Group. Transitions of care measures 2008 [on-line]. Available at http://www.ntocc.org/Portals/0/PDF/Resources/TransitionsOfCare_Measures.pdf Accessed August 15, 2012.

43. Moher D, Liberati A, Tetzlaff J et al. Preferred reporting items for systematic reviews and meta-analyses: The PRISMA statement. PLoS Med 2009:e1000097. 
